Cipango Cahiers d'études japonaises

23 | 2020

Charbon et communautés minières au Japon

\title{
Le soutien aux travailleurs déplacés dans le déclin de l'industrie houillère japonaise : un soutien officiel et associatif
}

Support for Displaced Workers in a Context of Decline of the Japanese Coal Industry: Official and Associative Support

\section{Naoko Shimazaki}

Traducteur : Bernard Thomann

\section{(2) OpenEdition}

\section{Journals}

Édition électronique

URL : https://journals.openedition.org/cipango/3746

DOI : $10.4000 /$ cipango.3746

ISSN : 2260-7706

Éditeur

INALCO

Édition imprimée

Date de publication : 1 juillet 2020

ISBN : 9782858313426

ISSN : 1164-5857

Référence électronique

Naoko Shimazaki, «Le soutien aux travailleurs déplacés dans le déclin de l'industrie houillère japonaise : un soutien officiel et associatif », Cipango [En ligne], 23 | 2020, mis en ligne le 16 juin 2020, consulté le 30 juin 2021. URL : http://journals.openedition.org/cipango/3746 ; DOI : https://doi.org/ 10.4000/cipango.3746

\section{(c) (7) \&}

Cipango est mis à disposition selon les termes de la Licence Creative Commons Attribution - Pas d'Utilisation Commerciale 4.0 International. 


\title{
Le soutien aux travailleurs déplacés dans le déclin de l'industrie houillère japonaise : un soutien officiel et associatif ${ }^{1}$
}

\author{
Support for Displaced Workers in a Context of Decline of the \\ Japanese Coal Industry: Official and Associative Support
}

\author{
Naoko SHIMAZAKI \\ Université de Waseda \\ Traduit du japonais par Bernard ThOMANN
}

Au Japon, l'industrie houillère constitua un exemple typique de restructuration industrielle. Aux côtés de l'industrie sidérurgique, elle fut considérée comme un secteur-clé et une priorité par les politiques industrielles de la période de reprise économique qui suivit la guerre. Elle commença toutefois à faire l'objet de mesures de rationalisation après avoir connu un ralentissement de 1953 à 1954 : en 1955, fut promulguée la « Loi sur les mesures temporaires concernant la rationalisation des mines de charbon » (sekitan kōgyō görika rinji sochi-hō 石炭鉱業合理化

1. Cet article est la traduction d'une version remaniée, en accord avec l'auteur, de SHImAZAKi Naoko 嶋崎尚子, 2013, Sekitan sangyô no shûsokukatei ni okeru rikushokusha shien 石炭産業の収束過程における離職者支援, Nihon rôdô kenkyû zasshi 日本労働研究雑誌, nº 641, pp. 4-14. 
臨時措置法). Le grave ralentissement économique qui s'installa à compter de 1957 entraîna par la suite une vague de fermetures de mines. Le gouvernement japonais lança alors dès 1959 sa politique « mettre au rebut et développer » (sukurappu ando birudo スクラップ・アンド・ビルド), qui visait à réduire les coûts de production et à améliorer la capacité concurrentielle de l'industrie en établissant des distinctions entre les mines qui devaient être développées au moyen d'investissements gouvernementaux, et les mines qui devaient être laissées en l'état ou fermées rapidement ${ }^{2}$. À compter de l'introduction de la «Première politique charbonnière » (daiichiji sekitan seisaku 第一次石炭政策) en 1963, et jusqu'en 2002, l'industrie houillère japonaise devait connaître neuf changements de politique en l'espace de quatre décennies.

Ces changements de politique, qui s'inscrivaient dans un contexte de concurrence avec le pétrole et le charbon importés, étaient contemporains d'initiatives en faveur de la nationalisation et de la concentration des entreprises dans les industries houillères étrangères. Citons à cet égard la nationalisation de l'industrie houillère britannique, et l'introduction d'un soutien gouvernemental aux compagnies minières en Allemagne. Ce type de mesures n'a cependant pas été adopté au Japon : ce fut sous la gestion de sociétés privées, principalement des conglomérats issus des anciens zaibatsu, que l'industrie houillère japonaise périclita dans le cadre d'un long processus de restructuration en réponse à l'évolution de la structure de l'offre énergétique ${ }^{3}$. De 1955 à 2002, 928 mines de charbon furent fermées et plus de 200000 travailleurs perdirent leur emploi. À l'heure actuelle, la seule mine extrayant encore du charbon est la Kushiro Coal Mine Co Ltd, où des ingénieurs étrangers en visite sont par ailleurs formés dans le cadre des programmes internationaux de transfert de technologies menés par le Japan Coal Energy Center (JCOAL) - programmes prévoyant également l'envoi d'ingénieurs experts japonais dans les pays participants.

Ce fut en réalité dès la fin de la guerre de Corée, en 1953, que les problèmes de chômage et de pauvreté des travailleurs licenciés des mines de charbon

2. KoYŌ SOKUSHIN JigYōdAN, 1992, p. 125.

3. Pour une analyse plus détaillée du processus de restructuration de l'industrie houillère au Japon, voir Culter, 1999, et ShimaZaki, 2010. 
s'aggravèrent dans les bassins houillers, en particulier dans celui du Chikuhō, sur l'île de Kyūshū $\bar{u}^{4}$. Ces questions constituèrent une toile de fond à la promulgation en 1959 de la « Loi sur les mesures temporaires en faveur des travailleurs des mines de charbon ayant perdu leur emploi »(tankō rishokusha rinji sochi-hō 炭 鉱離職者臨時措置法). Il s'agissait d'une 《 loi spéciale prévoyant des mesures globales et à long terme pour les travailleurs des mines de charbon licenciés, compte tenu de la nature structurelle du ralentissement de l'industrie houillère, de la forte concentration de travailleurs des mines de charbon licenciés dans certaines collectivités locales et des difficultés liées aux reclassement nécessitant un déménagement. ${ }^{5} \gg$ Cette loi était en l'occurrence un engagement à dépasser les mesures jusqu'alors poursuivies (des « mesures palliatives visant en grande partie à absorber les mineurs sans emploi dans des initiatives et projets publics de lutte contre le chômage »), et à fournir des « mesures globales aux travailleurs des mines de charbon déplacés », y compris de la formation professionnelle, des services d'assistance, et des placements à l'échelle la plus large du territoire ${ }^{6}$.

En 1963, la « Loi sur les mesures temporaires pour les travailleurs des mines de charbon déplacés » fut amendée. Un cadre fut alors élaboré pour faire en sorte que les titulaires de droits miniers soient obligés de fournir des mesures d'aide à l'emploi ${ }^{7}$, avec mise en place d'un système d'assurance chômage spécial pour les

4. Pour un compte rendu détaillé de la situation des chômeurs issus des petites et moyennes mines du Chikuhō entre 1954 et 1958, voir Yoshimura, 1984. Des informations supplémentaires sur la hiérarchie du marché du travail dans le Chikuhō peuvent être trouvées chez SMITH, 2005.

5. KoYō SOKUSHIN JIGYōDAN, 1992, p. 128.

6. Ibid., p. 129.

7. L'article 7 stipule : « Les titulaires de droits miniers [...] doivent coopérer avec l'Agence publique pour la sécurité de l'emploi et la Société de promotion de l'emploi afin de prendre les mesures nécessaires au développement des offres d'emploi et fournir d'autres formes d'aide à la recherche d'emploi, ceci dans le but de promouvoir les possibilités d'emploi pour les travailleurs des mines de charbon contraints de quitter leur emploi en raison de procédures de rationalisation de l'industrie minière, telles que la cessation d'une exploitation minière ou la modernisation de puits de mines. » 
mineurs, dit 《système du carnet noir 》 (kurotechō seido 黒手帳制度) ${ }^{8}$. Celui-ci allait rester en service pendant environ quarante ans, jusqu’à ce que la « Loi sur les mesures temporaires en faveur des travailleurs des mines de charbon ayant perdu leur emploi » soit abrogée en 2002.

Il importe également de noter, même si nous n'aborderons pas ce point plus en détail dans notre article, que cet ensemble de mesures pour les travailleurs des mines de charbon licenciés venait s'ajouter au régime de retraite particulier de l'industrie du charbon. Dans ce dernier cadre, les propriétaires de houillères prenaient à la fois en charge le coût des primes (soient 70 yens par tonne de charbon produite) et le fonds de pension des mines de charbon, créé en 1967 pour verser aux travailleurs retraités un complément à la retraite ordinaire. Notons par ailleurs dans le secteur minier, l'âge minimal requis pour commencer à bénéficier de prestations de retraite était moins avancé que dans le reste de l'industrie, ce qui eut pour effet de circonscrire quelque peu l'étendue du problème des mineurs âgés ayant perdu leur emploi.

Bien que l'industrie houillère japonaise n'ait pas été placée sous le contrôle de l'État, la question des travailleurs licenciés des mines de charbon fut davantage reconnue comme un problème à traiter au niveau national qu'une question spécifique à quelques entreprises ou bassins houillers. Les mesures de soutien furent donc tenues pour des initiatives nationales d'une importance stratégique pour les intérêts de l'ensemble de la société, et 4000 milliards de yens de financements publics furent injectés dans cette action en faveur de l'industrie houillère et des travailleurs licenciés ${ }^{9}$. L'ampleur et l'importance de l'industrie houillère au Japon, ainsi que la forte concentration de chômeurs dans ces communautés locales, constituent deux des principaux facteurs qui conduisirent le gouvernement à mettre en place un dispositif d'une telle envergure. Suganuma Takashi souligne trois autres facteurs politiques et économiques ${ }^{10}$ - à commencer par le poids traditionnel du mouvement syndical dans l'industrie houillère, de telles mesures ayant pour but d'éviter l'agitation sociale. Au cours

8. Rōdōshō Shokugyō anteikyoku, 1971, p. 209.

9. Shimanishi, 2011 , p. 7.

10. Suganuma, 1997, p. 38-39. 
$\mathrm{du}$ processus de redressement du Japon après la guerre, l'industrie houillère avait par ailleurs été redynamisée dans le cadre d'un contrôle étatique fort, et le gouvernement s'était en quelque sorte retrouvé responsable de la question de l'emploi dans cette branche industrielle. Enfin, la prise de conscience du ralentissement conjoncturel provoqué par l'évolution structurelle de la demande énergétique internationale joua également un rôle dans la mise en place de ces mesures. La crise de l'industrie houillère japonaise fut ainsi interprétée comme une crise industrielle inévitable due à des facteurs extérieurs. On estima par conséquent que cette situation dépassait la capacité de gestion des employeurs, syndicats et autres parties concernées, appelant inévitablement une intervention sous forme de politiques publiques.

Le présent article analysera à la lumière des processus de restructuration de l'industrie houillère évoqués supra deux questions : comment, d'une part, les travailleurs des mines de charbon déplacés retrouvèrent un emploi dans d'autres entreprises et industries, et comment les mesures de soutien à l'emploi qui leur furent proposées les aidèrent dans cette opération. Deux faits majeurs ressortent de leur parcours : bien que le système d'assurance-chômage pour les mineurs déplacés ait été mis au point afin d'apporter une aide à ces populations, force est de constater que ce système dit du « carnet noir » se révéla insuffisant. Ce fut un soutien plus personnalisé, faisant notamment appel au sens traditionnel de la solidarité entre les mineurs, qui contribua à compenser les insuffisances des mesures de soutien plus institutionnelles.

\section{Relocalisation et soutien aux travailleurs des mines de charbon licenciés}

\section{Reclassement et relocalisation}

Les travailleurs des mines de charbon ayant perdu leur emploi suite aux mesures de rationalisation et de fermeture présentaient trois caractéristiques distinctes. Tout d'abord, ils se concentraient dans des régions périphériques du Japon, caractéristique héritée de la naissance de l'industrie houillère. Deuxièmement, il était difficile pour les travailleurs licenciés de changer de secteur d'emploi. Enfin, leur perte de travail entraînait aussi la destruction des fondations sur lesquelles leur famille s'était construite. Les travailleurs déplacés n'avaient pas d'autre 


\section{CIPANGO \\ 180 \\ Cahiers d'études japonaises $n^{\circ} 23$}

choix que de trouver un autre type d'emploi, ce qui impliquait de se réinsérer dans la société tout en se déplaçant dans une nouvelle région. La relocalisation d'un grand nombre de travailleurs était susceptible de provoquer l'effondrement de communautés régionales entières, avec des conséquences non seulement économiques, mais aussi sur l'éducation des enfants et le bien-être des personnes âgées.

Comme nous l'avons souligné plus haut, il était difficile pour les travailleurs miniers déplacés de chercher un emploi dans d'autres industries. Cet état de choses était en partie dû à la nature particulière des compétences techniques qu' ils avaient acquises dans l'extraction du charbon et, dans le cas des mineurs travaillant à l'intérieur des puits de la mine, au salaire relativement élevé qu'ils percevaient à ce poste. Lors des enquêtes de terrain que nous avons menées, cinq tendances se sont en outre dégagées des souhaits exprimés par les travailleurs des mines de charbon à la recherche d'un emploi : nombreux sont ceux qui souhaitaient, dans l'ensemble, rester là où ils ont grandi ; les plus jeunes aspiraient à trouver un emploi rapidement ; les plus âgés souhaitaient vivement être employés par d'autres mines de charbon ; la plupart désiraient vivre dans un logement fourni par leur nouveau lieu de travail ; un grand nombre, encore une fois principalement les plus jeunes, souhaitait enfin recevoir une formation en vue de sa réintégration professionnelle ${ }^{11}$.

Compte tenu des caractéristiques des mineurs et de leurs aspirations, cinq types d'entreprises ou entités analogues pouvaient constituer des points de chutes professionnels crédibles. Premièrement, les « deuxièmes entreprises » (dainikaisha 第二会社) ou 《secondes mines » (dainitankōo 第二炭鉱), c'est-àdire les sociétés établies dans le but de donner une seconde vie à une mine fermée, qui ont joué un rôle important dans le réemploi des travailleurs déplacés depuis la période de la politique « mettre au rebut et développer » (1959-1967) jusqu’à celle de la «contraction équilibrée » (shukushō kinkō 縮小均衡, 1968-1972). Ces sociétés, qui offraient toutefois de faibles perspectives d'avenir, créèrent de surcroît une inadéquation de l'offre et de la demande : les travailleurs âgés qui restaient dans leur ville d'origine souhaitaient en effet être employés par ces

11. ShimAZAKi, 2012. 
entreprises pour éviter d'avoir à changer d'industrie, tandis que les employeurs préféraient manifestement employer des personnes plus jeunes dans le but de renforcer les opérations.

La deuxième catégorie d'entreprises susceptibles d'embaucher ces travailleurs sans emploi étaient les mines de charbon. Comme nous le verrons infra, de nombreux efforts furent déployés à Hokkaidō pour trouver un nouvel emploi minier aux travailleurs des houillères : ce « retour au travail minier »(tankō fukki 炭鉱復帰) était en effet considéré comme une priorité devant la pénurie chronique de main-d'œuvre qui touchait les mines bientôt agrandies par les investissements du gouvernement. Créée en 1966 sous l'étiquette de « mine à développer », la mine Mitsubishi de Minami Oyūbari 南大夕張炭鉱 fut lancée de la sorte, en employant des mineurs de charbon licenciés suite à la fermeture d'autres mines. Au plus fort de sa prospérité, 1540 personnes travaillaient sur ce site minier.

Les filiales offraient également une opportunité de réemploi aux travailleurs licenciés. Ces entreprises étaient le résultat de la diversification ou de la transformation des grands groupes miniers. Les filiales établies dans les villes minières, en particulier, furent les principaux lieux d'accueil des travailleurs déplacés dans la région même. Dans le cas des conglomérats issus des zaibatsu, il était également possible d'intégrer des travailleurs dans les entreprises du même groupe.

Le quatrième type de débouché, qui joua un rôle central, se situait dans les autres industries. Dans le cadre des politiques d'aide aux bassins houillers en difficulté, des efforts avaient été déployés pour attirer des entreprises, qui ne rencontrèrent toutefois que peu de succès. Par conséquent, la plupart des programmes de réemploi furent pilotés par des services de placement opérant sur des zones très étendues. Le réemploi impliquait donc inévitablement la réinstallation dans une autre région, et un nombre important de travailleurs furent déplacés du Kyūshū vers les régions du Kinki (départements d'Ōsaka et de Hyōgō) et du Chūbu (département d'Aichi), ainsi que des bassins miniers de Hokkaidō et de Jōban vers les zones industrielles de la conurbation de Tōkyō. Les efforts visant à développer les débouchés dans les autres industries consistèrent également à encourager activement les entreprises à embaucher collectivement des travailleurs déplacés par des recrutements massifs, que ce soient des travailleurs 


\section{CIPANGO}

182 Cahiers d'études japonaises $n^{\circ} 23$

âgés, des cols blancs, ou de jeunes travailleurs. Ce type de réemploi signifiait cependant pour les travailleurs déplacés une reconversion professionnelle et une relocalisation dans une autre région, mais aussi le défi particulier de l'adaptation à la vie urbaine. Il rencontra donc de fortes réticences de la part des mineurs. L'une des solutions proposées pour résoudre ce problème était donc que les travailleurs soient réemployés en groupe.

La cinquième option pour les travailleurs licenciés consistait à suivre une formation professionnelle afin de se préparer aux impératifs des mutations industrielles et des reclassements décrits plus haut. Certains travailleurs suivaient également une formation sur la base d'une promesse d'emploi dans une entreprise d'une autre branche. De nombreux travailleurs, en particulier les jeunes, demandèrent à bénéficier de tels dispositifs.

Dans ce contexte, et dans le cadre du système du « carnet noir », les travailleurs licenciés réintégrèrent le marché du travail avec un certain succès au cours de la période qui leur permettait de bénéficier de prestations d'assurance-chômage ${ }^{12}$ puis de promotion de l'emploi, soit un total de trois années de versement. Le résultat peut être observé à partir des statistiques sur les travailleurs licenciés dans chaque région ${ }^{13}$. Bien entendu, la capacité des travailleurs à trouver un nouvel emploi variait selon le bassin houiller, le moment de la fermeture de la mine de charbon, les caractéristiques de l'employeur et les caractéristiques individuelles de chaque travailleur.

12. Ibid. La durée de versement des prestations d'assurance-emploi était initialement de 270 jours, mais fut prolongée par la suite. Dans le cas des mines Ikeshima et Taiheiyō, qui furent les deux dernières mines à fermer, elle fut même portée à 330 jours, reflétant la gravité de la situation pour les travailleurs licenciés. En fait, la possibilité pour les travailleurs déplacés de trouver un nouvel emploi différait considérablement selon le moment de la fermeture du site qui les employait jusqu' ici.

13. Depuis le lancement du système du « carnet noir » au cours de l'année fiscale 1962 , et jusqu'à l'année fiscale 1970, la tendance au réemploi des travailleurs déplacés des mines de charbon fut telle que 83,9\% des 116250 travailleurs concernés étaient entrés dans l'industrie secondaire, dont $72,5 \%$ dans l'industrie manufacturière et 7,9\% dans celle de la construction. $53 \%$ des travailleurs avaient déménagé hors des régions charbonnières, le pourcentage le plus élevé $(25,6 \%)$ ayant été relocalisé dans le département d'Aichi. Rōdōshō SHokugyō anteikyoku, 1971, p. 341, tableau 4-20. 
Par exemple, la fermeture en 1971 de la mine de charbon Jōban (Jōban tankō 常磐炭鉱, ville d'Iwaki, département de Fukushima) entraîna le licenciement le plus massif observé dans l'industrie houillère au Japon : 4702 personnes se retrouvèrent en une seule fois sans emploi. $91 \%$ des 4171 personnes qui cherchèrent un nouvel emploi (soit un total de 3804 personnes) avaient retrouvé une place dans les seize mois suivant leur licenciement ${ }^{14}$. Parmi elles, 993 personnes (24\%) avaient trouvé un emploi à la mine Seibu, la « deuxième entreprise » créée après la fermeture, et 2612 personnes (63\%) avaient été embauchées dans d'autres industries. Nombre de ceux qui intégrèrent ces industries rejoignirent des sociétés affiliées dans la région, quand 1115 personnes (27\%) déménagèrent en dehors du département de Fukushima, principalement à Tōkyō, Chiba et Kanagawa.

TABLEAU 1. NOMBRE DE TRAVAILLEURS LICENCIÉS EN RAISON DE LA FERMETURE DE MINES DE CHARBON ET STATISTIQUES DE RÉEMPLOI.

\begin{tabular}{|c|c|c|c|c|c|c|}
\hline $\begin{array}{l}\text { Nom de la } \\
\text { mine }\end{array}$ & $\begin{array}{c}\text { Date de } \\
\text { fermeture }\end{array}$ & Département & $\begin{array}{c}\text { Nombre } \\
\text { de tra- } \\
\text { vailleurs } \\
\text { licenciés }\end{array}$ & $\begin{array}{c}\text { Nombre } \\
\text { de tra- } \\
\text { vailleurs } \\
\text { licenciés } \\
\text { ayant } \\
\text { cherché } \\
\text { un } \\
\text { emploi }\end{array}$ & $\begin{array}{l}\text { Pourcentage } \\
\text { de réemploi }\end{array}$ & $\begin{array}{c}\text { Nombre } \\
\text { de mois } \\
\text { écoulés } \\
\text { entre le } \\
\text { licencie- } \\
\text { ment et le } \\
\text { calcul du } \\
\text { pourcen- } \\
\text { tage de } \\
\text { réemploi }\end{array}$ \\
\hline $\begin{array}{c}\text { Kaijima } \\
\text { 貝島 }\end{array}$ & Sept. 1966 & $\begin{array}{l}\text { Fukuoka } \\
\text { 福岡県 }\end{array}$ & 1923 & 1839 (a) & $91.7 \%$ & 9 \\
\hline $\begin{array}{l}\text { Yūbetsu } \\
\text { 雄別 }\end{array}$ & Fév. 1970 & $\begin{array}{c}\text { Hokkaidōo } \\
\text { 北海道 }\end{array}$ & 2328 & 1925 (a) & $96.8 \%$ & 12 \\
\hline $\begin{array}{l}\text { Jōban } \\
\text { 常磐 }\end{array}$ & Avril 1971 & $\begin{array}{l}\text { Fukushima } \\
\text { 福島県 }\end{array}$ & 4702 & $4171(\mathrm{~b})$ & $91.2 \%$ & 16 \\
\hline
\end{tabular}

14. Shimazaki, 2004, p. $43-56$. 


\section{CIPANGO}

184 Cahiers d'études japonaises $n^{\circ} 23$

\begin{tabular}{|c|c|c|c|c|c|c|}
\hline $\begin{array}{c}\text { Sumitomo } \\
\text { Ponbetsu } \\
\text { 住友奔 } \\
\text { 別 }\end{array}$ & Oct. 1971 & $\begin{array}{c}\text { Hokkaidō } \\
\text { 北海道 }\end{array}$ & 2335 & 2032 (a) & $69 \%$ & 5 \\
\hline $\begin{array}{c}\text { Sumitomo } \\
\text { Utashinai } \\
\text { 住友歌 } \\
\text { 志内 }\end{array}$ & Oct. 1971 & $\begin{array}{c}\text { Hokkaidō } \\
\text { 北海道 }\end{array}$ & 1124 & 943 (a) & $79.2 \%$ & 5 \\
\hline $\begin{array}{c}\text { Hokutan } \\
\text { Yūbari } \\
\text { tankō } \\
\text { 北炭夕張 } \\
\text { 新鉱 }\end{array}$ & Oct. 1982 & $\begin{array}{c}\text { Hokkaidō } \\
\text { 北海道 }\end{array}$ & 1905 & 1711 (b) & $57.6 \%$ & 3 \\
\hline $\begin{array}{c}\text { Mitsui } \\
\text { Miike } \\
\text { 三井三 } \\
\text { 池 }\end{array}$ & Mars 1997 & $\begin{array}{c}\text { Kumamoto } \\
\text { 福岡・熊本 }\end{array}$ & 1553 & 1317 (a) & $80.9 \%$ & 50 \\
\hline $\begin{array}{c}\text { Ikejima } \\
\text { 池島 }\end{array}$ & Nov. 2001 & $\begin{array}{c}\text { Nagasaki } \\
\text { 長崎県 }\end{array}$ & 1214 & 954 (a) & $49.7 \%$ & 35 \\
\hline $\begin{array}{c}\text { Taiheiyō } \\
\text { 太平洋 }\end{array}$ & Jan. 2011 & $\begin{array}{c}\text { Hokkaidō } \\
\text { 北海道 }\end{array}$ & 1066 & 1016 (b) & $70.7 \%$ & 36 \\
\hline
\end{tabular}

Notes : a. Cols blancs, mineurs réguliers, journaliers (salariés temporaires ou de soustraitants) ; b. Cols blancs, mineurs réguliers.

Sources : Kaishima : Takahashi, Takagawa (dir.), 1987 ; Yūbetsu : Hokkaidō tankō rishokusha koyō engo kyōkai, 1978 ; Jōban : Shimazaki, 2004 ; Sumitomo Ponbetsu et Sumitomo Utashinai : Hokkaidō tankō rishokusha koyō engo kyōkai, 1978 ; HokutanYūbari tankō : Ōba, 2011 ; Mitsui Miike : Kodamu, 2001 ; Ikejima : Hama, 2004 ; Taiheiyō : Shimazaki, Sutō (dir.), 2012. 


\section{Contenu des mesures de reclassement et de soutien}

Les politiques de fermeture des mines et les mesures à l'égard des travailleurs reclassés

Prendre en charge le reclassement de près de cinq mille travailleurs en peu de temps, comme ce fut le cas à la mine de charbon Jōban, représentait une tâche considérable. Les mesures alors proposées furent élaborées sur une longue période, dans le cadre d'un projet d'envergure impliquant non seulement la mine ellemême, mais aussi la communauté régionale dans son ensemble ${ }^{15}$.

La mine Jōban constitue à cet égard un cas d'école dans l'établissement de mesures de reclassement et de soutien aux travailleurs ${ }^{16}$. Après avoir fondé le «Centre d'Iwaki pour les mesures de reconversion » (Iwaki kōgyōsho tenshin taisaku honbu 磐城砿業所転進対策本部), les représentants syndicaux et patronaux commencèrent dès la mi-février 1971 à recueillir des renseignements sur les possibilités de réemploi des travailleurs en prévision de la fermeture du site, le 29 avril suivant. Le centre mena également une enquête auprès de tous les travailleurs qui devaient être licenciés quant à leur intention de rechercher un nouvel emploi. Dans le même temps, la ville d'Iwaki conçut en collaboration avec l'organisme gouvernemental de placement et de conseil en matière d'emploi, l'«Agence publique locale pour la sécurité de l'emploi »(Kōkyō shokugyō anteisho 公共職業安定所), un cadre pour créer de nouvelles opportunités d'emploi, offrir des services de conseil en orientation professionnelle et créer des perspectives professionnelles. Ce cadre fut effectif jusqu'à la fin des prestations d'assurance-chômage, en mai 1972. Les quatre missions majeures du centre d'Iwaki consistaient ainsi à garantir des offres de recrutement de masse, promouvoir l'emploi des travailleurs âgés, développer de nouvelles possibilités d'emploi dans les entreprises locales, et persuader les travailleurs d'accepter un réemploi en dehors du département.

Les mesures en faveur des travailleurs déplacés commencèrent à être mises en œuvre, comme nous l'avons souligné, dès le moment où la date de fermeture de la

15. Ibid., p. 4446

16. Pour davantage d'informations sur la mine de Jōban, voir SHimazaki, 2011. 
mine fut décidée, soit avant la conclusion de l'accord officiel de fermeture entre le syndicat et la direction. Par conséquent, le cadre était déjà en place au moment de la fermeture effective de la mine. Il s'agissait par ailleurs d'assurer le réemploi des travailleurs durant leur période de perception des prestations d'assurance chômage, un objectif qui put être atteint dans le cadre de la collaboration entre deux principaux acteurs. Le premier était l'exploitant de la mine, qui mit sur pied des services d'aide à l'emploi et de placement, ainsi que des « Comités de placement 》(Shūshokuassen iinkai 就職斡旋委員会) composés de représentants du syndicat et de la direction. Le second était l'administration locale du travail, c'est à dire l'Agence publique locale pour la sécurité de l'emploi, le « Bureau local de la Société de promotion de l'emploi » (Koyō sokushin jigyōdan shibu 雇用 促進事業団支部) et le 《Conseil de liaison des différents acteurs concernés 》 (Taisaku renraku kyōgikai 対策連絡協議会) ${ }^{17}$.

$\underline{\text { Le soutien individuel : orientation et aide au placement par les conseillers locaux }}$

Bien sûr, le problème de la réinsertion après le licenciement était avant tout celui du travailleur concerné, mais ceux-ci avaient besoin de conseils et d'un soutien spécifiques pour trouver un nouvel emploi. Il s'agissait principalement de faire correspondre les souhaits des travailleurs déplacés aux possibilités d'emploi.

Les initiatives visant à trouver de nouveaux emplois pour les travailleurs licenciés furent lancées le 5 mai 1971, peu après la fermeture de la mine. À cette époque, le nombre d'offres d'emploi identifiées s'élevait à 11250 , réparties entre 548 entreprises. Le nombre d'opportunités offertes par les entreprises locales était toutefois relativement limité, avec 2852 emplois proposés dans seulement 114 entreprises, y compris des sociétés affiliées. Pour cette raison, les différents acteurs impliqués poursuivirent leurs efforts en matière de conseil et de placement ${ }^{18}$.

17. La situation de la fermeture de la mine Kaijima en 1970 est présentée en détail dans TAKAGAWA \& TAKAHASHI, 1987.

18. Pour plus de détails, voir Shirai, 2001, p. 87-129. 
Si l'on examine le résultat de ces efforts en termes de réemploi, le nombre de travailleurs embauchés s'éleva à 1457 personnes. Onze sociétés embauchèrent au moins vingt salariés chacune, mais la plupart de ces travailleurs, soit 993 personnes, fut engagée par la mine de charbon Seibu, une « deuxième mine » nouvellement créée. Trois autres entreprises embauchèrent respectivement 100, 72 et 59 personnes. Sur ces 1457 placements, 464 personnes trouvèrent enfin un emploi hors département.

En plus de l'aide financière qu'ils percevaient (principalement sous forme d'allocations de chômage), les travailleurs licenciés reçurent par ailleurs une aide individuelle de retour à l'emploi et de réinstallation. Dans ce cadre, un certain nombre d'activités furent organisées : des séances d'information sur des sujets tels que l'assurance-chômage, les débouchés professionnels possibles (avec présentation d'entreprises et séances d'orientation), ainsi que des visites dans les établissements recruteurs. Un soutien individuel fut fourni à ces personnes par le biais, notamment, de « cartes d'orientation professionnelle » (shüshoku sōdan kâto 就職相談カート) destinées à déterminer leurs souhaits; à l'aide des renseignements recueillis dans ces cartes, des entretiens étaient ensuite menées avec eux pour leur présenter et leur expliquer les différentes possibilités d'emploi. Dans de nombreux cas, les femmes des travailleurs licenciés participaient également aux entretiens. Les travailleurs et leurs éventuelles épouses demandaient des renseignements et conseils à propos de sujets très variés, comme les salaires et les allocations d'emploi, les questions relatives au logement et à l'école de leurs enfants, les problèmes concernant un parent âgé, ou les possibilités d'emploi ouvertes à leurs épouses après le déménagement.

Lorsqu'il fallait prendre une décision finale quant à leur nouveau point de chute professionnel, de nombreux travailleurs avaient nécessairement besoin d'être aiguillés, n'ayant pas de critères sur lesquels fonder leur jugement. Les personnes impliquées dans la mise en place de ce soutien ont pu décrire leur situation comme suit : « peu de travailleurs disposaient de critères leur permettant de porter des jugements éclairés. Nombre d'entre eux ont donc fini par avoir du mal à prendre une décision, estimant qu'ils n'avaient pas d'autre choix que de se fier à l'opinion d'une personne influente qui leur était proche, à la recommandation du conseiller de l'Agence publique locale pour la sécurité de l'emploi, ou à l'avis 


\section{CIPANGO}

188 Cahiers d'études japonaises $n^{\circ} 23$

du Comité de placement, voire du syndicat de leur mine ${ }^{19}$. $\gg$ Ces pourvoyeurs de soutien se heurtèrent néanmoins à certaines difficultés :

Si l'Agence publique locale pour la sécurité de l'emploi fournit aux travailleurs déplacés et à tous les chômeurs des conseils en matière d'emploi, cette aide suppose qu'ils aient la « volonté et la capacité de travailler », condition sine qua non pour recevoir des prestations de chômage. Mais il est ardu de fournir une telle orientation à certains travailleurs venus demander de l'aide en raison de leurs difficultés à s'exprimer, difficultés caractéristiques des travailleurs des mines de charbon. Les travailleurs déplacés se méfient de l'orientation professionnelle fournie par l'Agence, des jugements qu'elle pourrait porter sur leur volonté de retour à l'emploi, et craignent que leurs allocations de chômage ne cessent d'être versées. Ils ont alors l'impression d'être confrontés à une organisation brutale, dont ils doivent se méfier. Dans de telles circonstances, il leur est difficile de choisir le bon emploi, ce qui affecte leur réinsertion ${ }^{20}$.

Les conseillers sur le terrain ont donc joué un rôle crucial en servant d'intermédiaire entre les travailleurs licenciés et l'Agence publique locale pour la sécurité de l'emploi. Employés mandatés par la Société de promotion de l'emploi, une agence créée par le gouvernement national, ces conseillers étaient chargés d'un large éventail de tâches, principalement des séances d'orientation pour les travailleurs sans emploi et les travailleurs nouvellement réembauchés ${ }^{21}$. S'ils étaient choisis sur la recommandation du syndicat, ces tâches pouvaient dans certains cas être confiées aux responsables syndicaux eux-mêmes. Comme

19. TAKagawa \& Takahashi, 1987, p. 103

20. OKUDA, 1992, p. 437.

21. En plus d'assurer ces séances, les conseillers locaux avaient un certain nombre de missions à accomplir : assurer à divers égards la liaison entre les demandeurs d'emploi et le Bureau de la sécurité d'emploi public, répondre aux demandes de conseils concernant la réinstallation, etc. KoYŌ SOKUSHIN JiGYŌDAN, 1992. 
ils n'étaient plus salariés de la mine de charbon (ou responsables syndicaux) à plein temps, mais restaient d'anciens mineurs bien connus des travailleurs, leurs conseils se montraient rassurants pour les personnes incertaines de la direction à prendre après la fermeture de leur mine.

Ces conseillers fournirent également un soutien aux travailleurs qui déménageaient vers un nouveau lieu de travail. On peut prendre l'exemple du rapport rédigé par un conseiller qui s'occupa en 1976 des travailleurs déplacés de la mine Kaijima Ōnoura 貝島大之浦 (Kyūshū), en tant que membre du service de placement de la mine ${ }^{22}$. Ce rapport note que les membres du service de placement et les conseillers locaux fournirent ensemble tout le soutien nécessaire à la migration d'un grand nombre de travailleurs (en prenant notamment des dispositions pour que les compagnies de chemin de fer accroissent le nombre de liaisons), ainsi qu'à l'accompagnement des travailleurs qui déménageaient seuls vers leur nouveau lieu de travail. En une semaine à peine, ce fut une centaine de ménages qui, grâce à leur action, émigrèrent de Kyūshū à Tōkyō, Ōsaka et autres endroits.

L'un des facteurs expliquant l'adoption et l'efficacité de ces mesures de soutien est la solidarité qui liait traditionnellement les communautés minières auxquelles ces mesures s'adressaient, une solidarité s'inscrivant à la fois dans le cadre fourni par l'entreprise, et dans celui du syndicat. L'esprit de communauté qui régissait tous les pans du quotidien des travailleurs et de leurs familles était illustré par des slogans tels que « la camaraderie de la mine » (yama no nakama ヤマの) 仲間), ou 《 une mine, une famille (ichizan ikka 一山一家), qui inspirèrent fortement les mesures adoptées pour soutenir les travailleurs licenciés ${ }^{23}$.

\section{$\underline{\text { Le soutien et le suivi des travailleurs réembauchés }}$}

L'objectif à court terme de l'aide aux mineurs licenciés était de leur permettre de retrouver un emploi et de faciliter la potentielle délocalisation qui pourrait s'ensuivre. Mais à long terme, il était également de garantir que les travailleurs

22. Takagawa \& Takahashi, 1987, p. 102

23. ICHIHARA, 1997, p. 371. 
puissent s'intégrer à leur nouvel environnement professionnel. Pour ce faire, il était nécessaire de leur fournir un soutien « de suivi » après le déménagement. Ce suivi comprenait des visites de travailleurs juste après leur reprise d'emploi pour vérifier qu' ils n'avaient pas de problèmes, ainsi que le traitement des plaintes, ou des conseils sur la façon de se réinstaller. Puis l'on rendait de nouveau visite aux travailleurs une année environ après leur entrée en fonction. Leur nouvelle entreprise devait en outre rendre des comptes si les travailleurs avaient été licenciés ou avaient vu leur statut modifié après l'expiration de la subvention à la stabilisation de l'emploi qui lui avait été versée. Dans les faits, un nombre considérable de travailleurs se retrouvèrent de nouveau au chômage au bout d'un an.

Considérées comme une partie importante des fonctions des différents services impliqués dans le replacement professionnel ${ }^{24}$, les enquêtes de suivi étaient menées par des employés de ces services et des syndicats. Par exemple, à la mine de charbon Shakubetsu 䣋別炭鉱 (Hokkaidō), une visite de suivi à grande échelle fut effectuée quatre mois après la fermeture du site, en 1970. Selon le rapport de visite, rendu public dans une parution commémorant la dissolution du syndicat, deux responsables syndicaux visitèrent sur une période de vingt jours dix mines de charbon et quinze sites de type industriel à Hokkaidō, puis dans dix autres départements. Le but de cette visite était d'encourager les travailleurs réembauchés, de mener des enquêtes pour vérifier leur situation depuis leur retour sur le marché du travail, de traiter des questions administratives, ou de fournir d'autres tâches de conseil. D'après le compte rendu, les anciens travailleurs éprouvaient le sentiment d'être perdus sur leur nouveau lieu de travail, et les représentants syndicaux en visite réagirent en exprimant leur compréhension et leurs encouragements.

Les entretiens que nous avons nous-mêmes menés confirment à quel point ces visites de suivi étaient rassurantes pour ceux qui avaient déménagé. Vingt et un ans après son déménagement, un ancien mineur interrogé se souvenait encore de deux conseillers lui ayant rendu visite à plusieurs reprises après son départ d'une grande mine de charbon à Hokkaidō vers la banlieue de

24. Takagawa \& Takahashi, 1987, p. 107-108. 
Tōkyō, en 1992. Ce travailleur avait été réembauché comme salarié régulier dans une entreprise manufacturière de la banlieue de la capitale avec deux de ses amis. En déménageant, il avait laissé sa famille à Hokkaidō et s'était installé dans le dortoir de la société. En mars suivant, il avait emmené sa famille vivre avec lui à Tōkyō et avait emménagé dans un logement géré par la Société de promotion de l'emploi. Étant donné que la période maximale d'occupation de ce logement était fixée à dix années, il avait acquis plus tard sa propre maison, dans laquelle il vit encore. Après avoir atteint l'âge de la retraite, il fut réembauché comme contractuel. Selon toutes apparences, il échangeait encore des cartes de vœux avec d'autres anciens mineurs et conseillers de la mine.

\section{Le soutien aux mineurs licenciés de Hokkaidō}

\section{La fermeture de mines à Hokkaidò et la création de l'Association d'aide à l'emploi pour les travailleurs sans emploi des mines de charbon d'Hokkaidö}

À partir des années 1960, le centre de gravité de l'industrie houillère se déplaça de Kyūshū à Hokkaidō, région qui devait en rester le pilier jusqu'à la fermeture des dernières mines. L'île septentrionale fut donc, sur une longue période, le lieu de nombreuses fermetures et mesures de rationalisation. L'aide aux travailleurs déplacés fut prise en charge par une association spécifique créée en 1968 : l'« Association d'aide à l'emploi pour les travailleurs sans emploi des mines de charbon de Hokkaidō » (Hokkaidō tankō rishokusha koyōengo kyōkai 北海道炭鉱 離職者雇用援護協会, ci-dessous 《Association d'aide 》). Cette organisation, chargée de contribuer au réemploi des travailleurs licenciés ou déplacés à la suite de la fermeture ou de la rationalisation des mines de Hokkaidō, s'attachait tout particulièrement à préparer le retour au travail des mineurs. L'Association d'aide apporta son soutien pendant trente-huit ans, jusqu'à sa dissolution en 2006. Juste après sa création, quatre-vingt mines de charbon et 39194 personnes nécessitaient déjà ses ressources ${ }^{25}$.

25. HOKKAIDŌ SEKITAN RISHOKUSHA KOYŌ ENGO KYŌKAI, 2006, p. 2. 
L'Association d'aide était une organisation associative, dont la branche régionale du «Syndicat des mineurs de charbon japonais » à Hokkaidō (Nihon tankō rōdō kumiai Hokkaidō shibu 日本炭鉱労働組合北海道支部) avait joué un rôle central dans la création. Au moment de sa formation, près de 800 mineurs âgés étaient restés sans aucune perspective de replacement dans un certain nombre de zones d'extraction du charbon; il était donc urgent de leur trouver un emploi. Les mineurs de charbon déplacés rencontraient de surcroît des difficultés pour s'adapter à la vie dans les zones urbaines, et se retrouvaient parfois impliqués, en particulier à Sapporo, dans des affaires de fraude.

D'après les travailleurs eux-mêmes, « les mineurs déplacés comptaient naturellement sur le Siège régional de Hokkaidō comme le seul endroit vers lequel se tourner, car il faisait partie du Syndicat des mineurs de charbon. » C'est pourquoi il se devait d'être une organisation fournissant aux travailleurs des « services de conseil » sur des questions variées, visant d'autre part à « mener des initiatives pour un soutien sérieux et sincère qui ne pouvait être fourni que par le secteur associatif ${ }^{26}$. 》

L'Association d'aide avait deux missions principales : fournir une assistance, et lancer des initiatives. Ses tâches en matière d'assistance comprenaient :

1. la poursuite des activités de soutien au réemploi ;

2. l'élaboration de mesures pour aider les travailleurs encore au chômage (et restés sans progrès au même endroit) à retrouver un emploi ;

3. la délivrance de conseils ;

4. le soutien aux travailleurs dans leurs démarches en matière de logement ;

5. le renforcement des cadres institutionnels de soutien;

6. la réalisation d'enquêtes (principalement de suivi);

7. des activités de communication.

Ces tâches correspondaient d'ailleurs aux principales responsabilités des services de mesures pour l'emploi susmentionnés, qui avaient été créés par les partenaires sociaux dans les autres bassins houillers. La première de ces missions

26. Ibid., p. 89. 
- aider à la réembauche des travailleurs déplacés - comportait des tâches d'orientation, de mise en œuvre de formations visant à promouvoir le réemploi, et des actions destinées à susciter de nouvelles offres d'emploi, aider les travailleurs à $s$ 'installer dans leur nouvel emploi, ou leur rendre visite pour les conseiller.

\section{Une relocalisation pour « un retour au travail minier 》}

L'aide au réemploi fournie par l'association visait principalement les travailleurs déplacés, qu'il s'agissait d'inciter à retourner dans les mines plutôt qu'à changer d'industrie. La situation des mines de charbon de Hokkaidō était quelque peu paradoxale : alors que les travailleurs d'un certain nombre de sites furent déplacés à la suite de fermetures et de mesures de rationalisation, un manque chronique de main-d'œuvre touchait les « mines à développer » dans le cadre de la politique «mettre au rebut et développer ${ }^{27} \gg$. L'un des principaux objectifs était de veiller à ce que les mines, en particulier les « mines à développer », continuent de fournir du charbon, tout en évitant les « fermetures de mines pour cause de manque de main-d'œuvre ».

Les statistiques concernant le réemploi fournies par l'Association d'aide et les mines montrent que le retour au travail minier joua un rôle important dans la réinsertion professionnelle. Par exemple, lors de la fermeture des mines Sumitomo Utashinai 住友歌志内 et Sumitomo Ponbetsu 住友奔別 en 1971, un total de 4716 personnes furent licenciées, dont 1661 à Utashinai et 3055 à Ponbetsu. Cinq mois après la fermeture de la mine, 2150 des 3459 personnes recherchant un nouvel emploi avaient été réembauchées. Parmi les 988 personnes qui étaient retournées travailler à la mine, 575 avaient été réemployées par une autre mine du groupe Sumitomo à Akabira 住友赤平, et les 413 restantes réaffectées dans

27. ICHIHARA, 1997, p. 371. 


\section{CIPANGO \\ 194 Cahiers d'études japonaises $n^{\circ} 23$}

quatorze autres mines et vingt-quatre sociétés sous-traitantes travaillant pour des mines $^{28}$.

Au moment de la fermeture de ces deux mines de Sumitomo, un « centre temporaire d'orientation professionnelle » (genchi rinji shokugyō sōgō sōdansho 現地臨時職業総合相談所) fut mis en place sur les lieux pour une période de cinq mois, et des séances organisées afin d'informer les travailleurs des aides disponibles. L'Association d'aide déploya également des efforts intensifs pour fournir un soutien, nommant un conseiller dans chaque mine.

Plutôt que d'être concentrés sur un même site, les mineurs qui avaient retrouvé un travail dans le charbon furent déplacés vers un grand nombre de mines différentes. En d'autres termes, les réaffectations se déroulèrent de manière non systématique, mais en fonction des souhaits des demandeurs d'emploi, ainsi que d'autres facteurs. Bien qu'il s'agît d'un « retour » au travail minier, il n'était pas simplement question d'échanges entre l'entreprise minière licenciant des travailleurs et l'entreprise houillère souhaitant en embaucher, mais bien d'un réemploi conforme aux procédures officielles de recrutement et de recherche d'emploi fondées sur le Système d'assurance chômage des mineurs sans emploi, ou « système du Carnet noir ». Il est néanmoins compréhensible, quoique légèrement paradoxal, que l'action de l'Association d'aide ait permis aux grands producteurs de charbon de Hokkaidō de poursuivre leur propre politique de rationalisation à partir de 1971 .

Les caractéristiques du soutien de l'Association d'aide à l'emploi pour les travailleurs sans emploi des mines de charbon d'Hokkaidō : un appui formel et informel

J'examinerai maintenant quatre points caractéristiques de l'assistance apportée aux mineurs de Hokkaidō, principalement par l'Association d'aide. Tout d'abord, la majorité des travailleurs qui furent déplacés dans la région de Hokkaidō, à

28. Ces chiffres sont ceux du 30 décembre 1971. Le nombre total de travailleurs qui retournèrent travailler dans une mine avait augmenté de trente-cinq personnes à la fin de mars 1972. 
l'exclusion des travailleurs qui réintégrèrent des mines, s'installèrent à Sapporo, la plus grande ville de l'île. Le soutien fourni par l'association d'entraide fut ainsi développé à partir de cette ville, ce qui explique pourquoi les initiatives visant à soutenir les travailleurs licenciés après la fermeture de la mine Taiheiyō à Kushiro, dans l'Est de Hokkaidō, aient été entravées par la distance même qui séparait de Sapporo cette mine, la dernière à avoir été fermée ${ }^{29}$. Pour l'association, il était essentiel de créer des perspectives d'emploi, mais aussi, entre autres tâches, de développer le logement. Une attention particulière fut ainsi accordée aux problèmes liés à l'éducation des enfants de mineurs suite à leur changement d'école, en particulier de lycée. Les conseillers étaient également au courant, dès le début, des problèmes concernant les parents âgés des travailleurs et les personnes âgées « laissées pour compte » dans les bassins miniers. Des efforts furent ainsi déployés pour encourager le gouvernement local de Hokkaidō à augmenter le nombre de soignants à domicile afin de venir en aide à ces personnes.

Deuxièmement, des permanences de conseil destinées aux anciens mineurs vivant à Sapporo furent assurées en continu, et ceux-ci demandèrent des conseils sur tous les aspects de leur vie quotidienne. Comme le siège régional du Syndicat des mineurs de charbon japonais l'avait proposé lors de la création de l'Association d'aide, les conseillers cherchaient à fournir aux travailleurs « un soutien sérieux et sincère, en les comprenant réellement par le biais de séances de conseil en face à face, lors desquelles ils pourraient partager leurs préoccupations, problèmes et heureux événements ${ }^{30} \gg$. À partir de la fin des années 1970, la récession économique entraina une augmentation du nombre de femmes au foyer sollicitant des conseils en matière d'emploi. Un traitement adéquat de ces demandes fut rendu possible, au cours des années fiscales 1978 et 1979, par l'affectation d'un conseiller aux questions concernant spécifiquement les femmes ${ }^{31}$. Ces populations de travailleurs purent également s'appuyer sur la collaboration de groupes formés par des anciens mineurs vivant à Sapporo, connus sous le nom d' « Associations

29. Entretien avec un ancien cadre supérieur de l'Association d'aide à l'emploi pour les travailleurs sans emploi des mines de charbon de Hokkaidō.

30. HoKKaIdō SEKITAN RISHOKUSHA KOYŌ ENGO KYŌKaI, 2006, p. 2.

31. HoKKaIdō SEKITAN RISHOKUSHA KOYō ENGO KYōKAI, 1988, p. 67. 
de la mine »(Yama no kai ヤマの会). À leur apogée, Sapporo compta jusqu’ à vingt-et-une Yama no kai, cependant toutes dissoutes en 2012, du fait du vieillissement de leurs membres. Le rôle central accompli par les Yama no kai dans le maintien du fort sentiment de solidarité qui liait entre eux les mineurs, et qui s'étendait jusqu'à leurs confrères restés dans les zones charbonnières, a contribué à compléter efficacement le soutien apporté par l'Association d'aide.

Troisièmement, des enquêtes auprès des travailleurs demeurés dans les zones minières (et autres enquêtes de suivi) furent également menées à intervalles régulières chaque année à partir de 1970, selon les archives. Afin d'étudier leurs conditions de vie réelles, 2000 individus furent sélectionnés parmi l'ensemble des travailleurs déplacés pour une enquête sur leur réemploi dans toutes les branches de l'industrie, principalement à Sapporo, dans un premier temps. Ces enquêtes s'attachèrent ensuite à suivre les travailleurs déplacés d'une mine spécifique, mais ne purent être menées à terme comme initialement prévu. Les archives révèlent par ailleurs qu'une enquête à propos des travailleurs résidant dans les logements de la Société de promotion de l'emploi fut conduite chaque année.

Un examen attentif de ces archives montre enfin que l'Association d'aide assuma des responsabilités considérables, mais qui finirent par rencontrer leurs limites. Suite au grave accident qui entraîna en 1982 la fermeture par le gouvernement de la mine Yūbari Shinkō 夕張新鉱, la plus prometteuse des « mines à développer », il lui apparut en effet difficile de fournir au nombre croissant de travailleurs demeurés dans leur région d'origine un soutien pour le retour au travail minier. Même si l'Association d'aide avait été formée par la collaboration entre employeurs et syndicats, il convient de préciser que le travail proprement dit fut effectué par le siège régional du Syndicat des mineurs de charbon japonais, sis à Hokkaidō.

\section{Conclusion : les effets et défis de l'aide à la réinsertion}

Au Japon, les processus de restructuration de l'industrie houillère se déroulèrent ainsi sur une longue période, durant laquelle un grand nombre de travailleurs des mines de charbon furent déplacés. Le gouvernement japonais reconnut que la réinsertion de ces travailleurs au sein de la société - y compris ceux qui commençaient une nouvelle carrière dans une industrie différente - était un 
problème pour l'ensemble du pays, par opposition aux problèmes spécifiques des entreprises privées et de chaque zone d'extraction du charbon. Il adopta ainsi une approche globale comprenant non seulement des mesures de réinsertion, mais aussi de réinstallation, de logement et de formation professionnelle. Il s'agissait de mesures et de systèmes de soutien substantiels, qui n'existaient pas dans les autres industries. Les problèmes fondamentaux des chômeurs restèrent cependant sans solution. Comme le montre la présente étude, l'aide officielle aux travailleurs licenciés reposait en réalité sur la capacité de mobilisation des entreprises individuelles et des collectivités locales, et présentait des caractéristiques particulières. Les insuffisances des systèmes de soutien officiels furent compensées par un soutien associatif reposant à la fois sur les relations interpersonnelles et le fort sentiment de solidarité entre collègues mineurs propres à la culture de l'extraction du charbon.

Divers éléments démontrent le rôle-clé joué par le soutien associatif, que nous pouvons résumer de la façon suivante : lorsque confrontée à une décision de fermeture, chaque mine bénéficiait non seulement d'un soutien financier gouvernemental, mais aussi d'un dispositif de soutien personnel individuel sous forme de conseils et d'une aide au placement des travailleurs qui changeaient d'industrie. Le fonctionnement de ce dispositif reposait sur les organes de placement professionnel publics, les associations, ainsi que des conseillers connaissant bien le terrain. Comme le montre clairement le cas de l'Association d'aide à l'emploi pour les travailleurs des mines de charbon déplacées à Hokkaidō, le syndicalisme joua un rôle central dans la mise en œuvre de ces mesures. Il faut néanmoins préciser que si le Syndicat des mineurs de charbon japonais, qui avait influencé le déroulement des conflits liés à la rationalisation, put lui aussi jouer un rôle de premier plan dans le soutien aux travailleurs licenciés, ce fut en raison de la défaite des syndicats d'entreprise dans les conflits ayant accompagné la fermeture des mines à Hokkaidō. Et c'est par le biais de cette fédération nationale que l'Association d'aide assuma à son tour un rôle central.

Le soutien à chaque travailleur déplacé prenait en compte les caractéristiques spécifiques de ces derniers. Il s'agissait en effet de remédier au manque de compétences socioculturelles des travailleurs miniers déplacés comme aux difficultés qu'ils rencontraient pour s'adapter à la vie en ville. Un moyen efficace de s'attaquer à ces problèmes consistait à mettre à profit la mentalité 
de « camaraderie entre mineurs » développée dans les conditions de travail difficiles et parfois dangereuses des houillères ${ }^{32}$. Aussi pouvons-nous affirmer que les systèmes de soutien, principalement ceux de l'Association d'aide, réussirent à s'accorder avec la culture ouvrière générée par les communautés minières.

Naturellement, les mesures et le soutien fournis aux travailleurs se heurtèrent à des problèmes et des limites, à commencer par la hiérarchie appliquée à la recevabilité du soutien. Les travailleurs déplacés ayant fait l'objet de cet article étaient des travailleurs miniers réguliers, directement employés, qui constituaient la majorité de la communauté minière. Il existait toutefois trois catégories de travailleurs dans ces mines de charbon : les employés « cols blancs », les mineurs réguliers, et le groupe de mineurs le plus faible au sein de cette hiérarchie : les journaliers (ou salariés sous-traitants). La condition de ces derniers était particulièrement médiocre par rapport à celle des mineurs directement employés, ce qui se vérifia également lors de la fermeture des mines. De nombreux journaliers ne pouvaient pas recevoir de « carnet noir » pour le système d'assurance chômage, et beaucoup n'étaient pas habilités à recevoir de prestations compensatoires du fonds de pension des mines de charbon. Ces travailleurs ne reçurent aucun des types de soutien décrits supra. En ce sens, les problèmes de chômage et de pauvreté qui avaient été constatés à Kyūshū avant l'adoption de la « Loi sur les mesures temporaires en faveur des travailleurs des mines de charbon déplacés » ne furent pas davantage résolus à Hokkaidō.

Les nouvelles possibilités d'emploi offertes aux cols blancs déplacés à la suite de fermetures étaient de surcroît généralement limitées, alors que ceux-ci jouissaient de conditions relativement favorables lorsqu'ils travaillaient encore dans les houillères. Dans le cas des conglomérats issus d'anciens zaibatsu, ces employés furent absorbés dans d'autres sociétés du groupe, voire parfois réembauchés avec d'anciens mineurs de charbon par le biais de processus de recrutements collectifs.

Le deuxième problème se situait au niveau de la durée d'accompagnement, garanti jusqu'au moment de la réembauche des mineurs, mais qui ne pouvait se

32. De plus amples informations à propos du sens de la solidarité et des caractéristiques particulières des mineurs de charbon au Japon peuvent être trouvées chez AlleN, 1994. 
prolonger que de douze mois maximum après leur retour à l'emploi. Le soutien accordé aux travailleurs par la suite congédiés de leur nouvel emploi se montra en outre insuffisant. Ne serait-ce qu'évaluer la situation professionnelle et personnelle des anciens mineurs de charbon déplacés se montrait même difficile une fois l'aide échue.

Enfin, la troisième difficulté provenait du fait que les mesures d'aide aux travailleurs déplacés avaient généralement été conçues à partir des entreprises qui licenciaient les travailleurs ; or, celles qui leur offraient un réemploi n'avaient pas établi de cadres de suivi et de soutien. Il était donc ardu pour certains travailleurs déplacés, qui avaient à la fois changé d'industrie et déménagé dans une nouvelle région, de s'intégrer à leur nouvelle communauté. Ceux qui avaient migré en nombre suite à une embauche collective - et commencé à vivre ensemble dans des logements de la Société de promotion de l'emploi avant de se disperser dans la population - risquaient tout particulièrement de se retrouver isolés.

Comme nous l'avons montré tout au long de cet article, le soutien apporté aux travailleurs et les mesures mises en place au cours de la longue restructuration de l'industrie houillère japonaise ont consisté en une aide à la fois globale et individuelle. Naturellement, il convient de noter que ce soutien et ces mesures furent réalisables dans le cadre très solidement enraciné de la négociation collective, doublé de la société centrée sur l'entreprise qui caractérisent le Japon de la seconde moitié du $\mathrm{XX}^{\mathrm{e}}$ siècle. Des mesures telles que celles-ci pourront même sembler incongrues dans le Japon d'aujourd'hui, où la flexibilité et la liberté du marché du travail se sont développées, et où les relations entre patronat et syndicats s'inscrivent davantage dans un mode individuel. Alors que nous sommes aujourd'hui confrontés à une période de transition structurelle de l'industrie, l'histoire exposée ici doit néanmoins nous rappeler que les travailleurs contraints de changer d'industrie n'ont pas seulement besoin de mesures temporaires contre le chômage, mais aussi de mesures globales offertes sur une période de temps raisonnable. Il est important de reconnaître la nécessité de telles mesures et de continuer à les développer. 


\section{CIPANGO \\ 200 Cahiers d'études japonaises $n^{\circ} 23$}

\section{Bibliographie indicative}

Allen Matthew, 1994, Undermining the Japanese Miracle. Work and Conflict in a Coalmining Community, Cambridge University Press, Cambridge, 290 p.

Culter Suzanne, 1999, Managing Decline: Japan's Coal Industry Restructuring and Community Response, University of Hawai'i Press, Honolulu.

Hama Tamio 浜民夫, 2004, Ikeshima tankō rishokusha no shūrō seikatsu jittai : Ikejima tankō rishokusha oyobi kazoku no shūrō seikatsu jittai ni kan suru chōsa bunseki heizan ninen o hete 池島炭鉱離職者の就労生活実態 : 池島炭鉱 離職者及び家族の就労・生活実態に関する調査分析 閉山 2 年を 経て [Les conditions réelles de vie au travail des travailleurs sans emploi de la mine d'Ikeshima : analyse des enquêtes sur les conditions de vie et de travail des anciens travailleurs de la mine d'Ikeshima et de leurs familles, deux ans après la fermeture], Nagasaki daigaku kankyō kagakubu rōdō kankyō kenkyūshitsu長 崎大学環境科学部労働環境研究室 [Centre de recherches de l'université de Nagasaki sur l'environnement du travail], Nagasaki.

HokKaidō SEKITAN RishokUsha KoYō ENGo KYŌKaI 北海道炭鉱離職者 雇用援護協会 [Association d'aide à l'emploi pour les travailleurs sans emploi des mines de charbon de Hokkaidō], 1988, Engo kyōkai nijūnen no ayumi kaisan resepushon 援護協会二十年の歩み解散レセプション [Réception commémorative de l'Association d'aide : vingt ans de vie associative].

Hokkaidō Sekitan Rishokusha Koyō Engo KyōKaI 北海道炭鉱離職 者雇用援護協会 [Association d'aide à l'emploi pour les travailleurs sans emploi des mines de charbon de Hokkaidō], 2006, Engo kyōkai jūnen no ayumi kaisan resepushon 援護協会十年の歩み解散レセプション [Réception commémorative de la dissolution de l'Association d'entraide : dix ans de vie associative].

Hokkaidō Sekitan Rishokusha Koyō Engo KyōKai 北海道炭鉱離職 者雇用援護協会 [Association d'aide à l'emploi pour les travailleurs sans 
emploi des mines de charbon de Hokkaidō], 2006, Engo kyōkai sanjūhachinen no ayumi kaisan resepushon 援護協会三十八年の歩夕解散レセプショ $\checkmark$ [Réception commémorative de la dissolution de l'Association d'entraide : trente-huit ans de vie associative].

ICHIHARA Hiroshi 市原博, 1997, Tankō no rōdō shakai-shi-Nihon no dentōteki rōdō shakai to chitsujo kanri 炭鉱の労働社会史一日本の伝統的労働社会 と秩序管理 [Une histoire sociale du travail dans les mines de charbon], Taga shuppan 多賀出版, Tōkyō.

Koyō Sokushin JigYōdan 雇用促進事業団 [Société de promotion de l'emploi], 1992, Koyō sokushin jigyōdan sanjūnenshi 雇用促進事業団三十 年史 [Trente ans d'histoire de la Société de promotion de l'emploi], Tōkyō.

Kusuda SeKitan Kōgyō 楠田石炭鉱業, 1952, « Rōdōsha no seikatsu jitai 》 in Rōdo tōkei chōsa geppōo 労働統計調査月報, $\mathrm{n}^{\circ}$ 4, vol. 4 .

OKUDA Haruo 奥田春男, 1992, 《Tankō rishokusha taisaku ni omou »炭 鉱離職者対策に思う [Réflexions sur les mesures pour les travailleurs des mines de charbon ayant perdu leur emploi], in Tanrō: Gekitō ano hi ano toki 炭労一激闘あの日あの時 [Le syndicat des mineurs de charbon du Japon : chronique d'une lutte acharnée], Nihon tankō rōdō kumiai, Tōkyō.

SAGAWA Kyōhei 佐川享平, 2015, « 1920 nendai no tankōgyō ni okeru gijutsu kakushin to rōdōryoku kōsei - Mitsubishi kōgyō chikuhō kōgyōjo ni okeru chōsenjin kōfu no shiyō kakudai o megutte » in Ōhara shakai mondai kenkyūjo $z a s s h i$ 大原社会問題研究所雑誌, $\mathrm{n}^{\circ} 677$.

ShImANASHI Tomoki 島西智輝, 2011, Nihon sekitan sangyō no sengoshi: Shijō $k \bar{o} z \bar{o}$ benka to kigyō kōdō 日本石炭産業の戦後史：市場構造変化と 企業行動 [Histoire de l'industrie charbonnière japonaise après la Seconde Guerre mondiale : les évolutions de la structure du marché et de l'activité des entreprises], Keiō gijuku daigaku shuppankai 慶應義塾大学出版会, Tōkyō. 


\section{CIPANGO}

202 Cahiers d'études japonaises n²3

SHimAZAKi Naoko 嶋㠃尚子, 2004, « Tankō rishokusha no saishūshoku kettei katei - Shōwa yonjūrokunen Jōban tankō KK daiheizanji no mikuro dēta bunseki » in Waseda Daigaku Daigakuin Bungaku kenkyūka kiyō 早稲田大 学大学院文学研究科紀要, $n^{\circ} 49$, pp. 43-56.

ShimaZaki Naoko 嶋㠃尚子, 2010, “Community and Workers in the Face of Coal Industry Decline" in actes de la conférence "Collective Memories of Coal Mining in Japan and Wales", université de Waseda.

SHImAZAKi Naoko 嶋㠃尚子, 2011, "History and Regeneration of Former Coal Mining Community, JOBAN in Japan" in actes de la conférence CTCC "On the Surface: The Heritage of Mines and Mining", université d'Innsbruck.

Shimazaki Naoko 嶋㠃尚子, 2011 actes de la conférence "Comparing Coalfields in Britain and Japan Symposium”, Gregynog, North Wales.

SHImAZAKi Naoko 嶋㠃尚子, 2012, «Santan chiiki kontekusuto to heizan rishokusha no saishūshoku katei: Chikuhō-Jōban-Kushiro » 産炭地域 コンテクストと閉山離職者の再就職過程＜筑豊・常磐・釧路 $>$ [Contextes et processus de réemploi des travailleurs ayant perdu leur emploi suite aux fermetures de mines de charbon dans le Chikuhō, à Jōban et à Kushiro] in Daihachijûkai Nihon shakai gakkai taikai hökoku 第 85 回日本 社会学会大会報告 [Rapport de la 85e conférence de l'Association japonaise de sociologie].

ShIRAI Chiaki 白井千晶, 2001, «Shūshoku taisaku honbu no taisaku to saishūshoku no suii » 就職対策本部の対策と再就職の推移 [Les mesures du Bureau d'action en faveur de l'emploi et les tendances en matière de réemploi], in MASAOKA Kanji 正岡寛司 (et al.) Tankō rōdōsha no heizan rishuku to kyaria no saikeisei 炭砿労働者の閉山離職とキャリアの再 形成一旧常磐炭矿KK. 矿員の縦断調査研究, Part III [Le déplacement des travailleurs des mines de charbon suite à la fermeture des mines et à la reconstruction des carrières : une étude longitudinale sur les mineurs de 
charbon de l'ancienne compagnie minière Jōban, $3^{\mathrm{e}}$ partie], Waseda daigaku Jōban tankō âkaibu kenkyū 早稲田大学常磐炭硹アーカイブ研究 [Groupe de recherches de l'université de Waseda sur les archives de la mine Jōban], Tōkyō, pp. 87-129.

Smith Donald W., 2005, "Gender and Ethnicity in Japan's Chikuho Coalfield”, in Berger Stefan, Croll Andy \& LaPorte Norman (eds.), Towards a Comparative History of Coalfiled Societies, Ashgate, Farnham, pp. 204-218.

Sone Sachiko, 2007, "The Reversible World of Japanese Coalmining Women" in The Australian Journal of Anthropology, vol. 18, pp. 207-222.

Suganuma Takashi 菅沼隆, 1997, « Tankō rishokusha taisaku » in Nihon rodō $k e n k y \bar{u} z a s s h i$ 日本労働研究雑誌, $\mathrm{n}^{\circ} 443$.

TAKAgaWA Masamichi 高川正通 \& TAKAhashi Shin'ichi 高橋伸一, 1987, «Sekitan kōgyō no seisui to rishokusha taisaku : Chikuhō, Kaijima tankō no jirei kenkyū » 石炭鉱業の盛衰と離職者対策一筑豊・貝島炭礦の 事例研究 [L'évolution de l'industrie houillère et les mesures en faveur des mineurs sans emploi : le cas de l'entreprise minière Kaijima dans le bassin de Chikuhō] in Bukkyō daigaku shakaigaku kenkyūjo kiyō 佛教大学社会学研 究所紀要 [Annales du Centre de recherches en sociologie de l'université Bukkyō], nº 8, pp. 81-109.

YAMAGUCHI Asuka 山口明日香, 2012, « Senji tōseiki no sekitan zōsan to shizai mondai », in Sugryama Shinya 杉山伸也 \& Ushijıma Toshiaki 牛島利 明 (eds.), Nihon sekitan sangyō no suitai 日本石炭産業の衰退, Keiō daigaku shuppankai 慶応大学出版会, Tōkyō. 


\section{CIPANGO}

204 Cahiers d'études japonaises n²3

Yoshimura Sakuo 吉村朔夫, 1984, Nihon tankōshi shichū 日本炭鉱史私注

[Commentaires sur l'histoire des mines de charbon japonaises], Ochanomizu shobō 御茶の水書房, Tōkyō.

Résumé : l'industrie houillère japonaise connut un déclin irréversible suite à de multiples vagues de restructuration. Ces mesures de restructuration résultèrent essentiellement de politiques réagissant à l'évolution de la structure de l'offre énergétique. À partir de 1955, 928 mines fermèrent, et plus de 200000 mineurs perdirent leur emploi. Les mesures en faveur de ces travailleurs licenciés, envisagées au niveau national, répondaient à des enjeux sociaux considérables. Ceci conduisit à la mise en place de systèmes de soutien substantiels qui n'existaient pas dans d'autres industries, pour prendre en compte non seulement le réemploi, mais aussi la relocalisation, le logement et la formation professionnelle. Les insuffisances des mécanismes de soutien institutionnel tendirent à être compensées par un soutien associatif fondé sur les relations interpersonnelles et le fort sentiment de solidarité entre collègues mineurs, caractéristiques de la culture ouvrière liée à l'extraction du charbon. L'aide aux travailleurs déplacés comprenait en effet non seulement une aide financière, mais aussi un soutien individuel, fournis par d'anciens travailleurs des mines agissant comme conseillers. Les syndicats jouèrent un rôle central dans l'élaboration de ces mesures.

Mots clés : mines de charbon, fermeture de mines, chômage, politique d'emploi, réemploi

Abstract: The Japanese coal industry experienced an irreversible decline following multiple waves of restructuring. These restructuring measures were mainly the result of policies responding to the changing energy supply structure. From 1955, 928 mines closed, and more than 200,000 miners lost their jobs. The measures in favor of these dismissed workers, considered at a national level, responded to considerable social challenges. This led to the establishment of substantial support systems which did not exist in other industries, to take into account not only re-employment, but also relocation, housing and vocational training. The inadequacies of the institutional 
support mechanisms tended to be compensated by associative support based on interpersonal relations and the strong feeling of solidarity among mining colleagues, characteristic of the workers' culture linked to coal mining. Assistance to displaced workers included not only financial assistance, but also individual support provided by former miners acting as advisors. Trade unions played a central role in the development of these measures.

Keywords: coal mining, shutting down of mines, unemployment, employment policy, reemployment

キーワード：石炭産業，炭鉱閉山，失業、雇用政策、再就職 
